\title{
Deriving Aerosol Parameters from in-situ Spectrometer Measurements for Validation of Remote Sensing Products
}

\author{
Sebastian Riedel ${ }^{* a, b}$, Joanna Janas ${ }^{\mathrm{b}}$, Peter Gege ${ }^{\mathrm{a}}$, Natascha Oppelt ${ }^{\mathrm{a}}$ \\ ${ }^{a}$ Kiel University Department of Geography, Ludewig-Meyn-Str. 14, 24098 Kiel, Germany; \\ ${ }^{\mathrm{b}}$ German Aerospace Center (DLR), Remote Sensing Technology Institute, 82234 Weßling, Germany
}

\begin{abstract}
Uncertainties of aerosol parameters are the limiting factor for atmospheric correction over inland and coastal waters. For validating remote sensing products from these optically complex and spatially inhomogeneous waters the spatial resolution of automated sun photometer networks like AERONET is too coarse and additional measurements on the test site are required. We have developed a method which allows the derivation of aerosol parameters from measurements with any spectrometer with suitable spectral range and resolution. This method uses a pair of downwelling irradiance and sky radiance measurements for the extraction of the turbidity coefficient and aerosol Angström exponent. The data can be acquired fast and reliable at almost any place during a wide range of weather conditions. A comparison to aerosol parameters measured with a Cimel sun photometer provided by AERONET shows a reasonable agreement for the Ångström exponent. The turbidity coefficient did not agree well with AERONET values due to fit ambiguities, indicating that future research should focus on methods to handle parameter correlations within the underlying model.
\end{abstract}

Keywords: Aerosol optical thickness; turbidity coefficient; Ångström exponent; spectrometer measurements; atmospheric correction; sun photometer; inland waters; field measurements.

\section{INTRODUCTION}

Atmospheric correction is a prerequisite for the application of quantitative evaluation methods to remote sensing data in optically complex coastal and inland waters. In particular when applying physically based bio-optical models, atmospheric correction is the limiting factor in the results accuracy [1]. The most critical parameter for atmospheric correction is the aerosol, especially for turbid and spatially inhomogeneous coastal and inland waters [2]. For validation of atmospheric correction accurate aerosol parameters, measured close to a number of matchup locations are required. Therefore it is often valuable to measure aerosol parameters during field campaigns on a boat. Aerosol parameters are usually derived from stationary instruments (self-aligning sun photometers [3] or rotating shadowband radiometers [4]) at fixed locations or from mobile handheld sun photometers [5] at a number of test sites during field campaigns. The spatial coverage of ground based sun photometers is often insufficient for validation purposes, and targeting the sun with the required accuracy below $1^{\circ}$ with a handheld device on a boat can be challenging [6]. To be more flexible during field campaigns, we have developed a method which allows the derivation of aerosol parameters from measurements with a field spectrometer. While sun photometers measure the radiance from the direction of the sun, this method uses downwelling irradiance and sky radiance measurements. Since no accurate alignment is necessary, the data can be acquired fast and reliable at almost any place during a wide range of weather conditions. In contrast to sun photometers and shadowband radiometers, spectrometers are commonly used by researchers in aquatic optics or limnology. Easy-toconduct field spectrometer measurements would therefore be a viable way to improve atmospheric correction procedures over inland and coastal waters.

\section{MATERIALS AND METHODS}

\subsection{Measurement Setup}

All measurements used in this paper were conducted with a Freedom VIS FSV-305 spectrometer from Ibsen Photonics [7]. The FSV-305 has a usable spectral range of 350-830 nm with a spectral resolution of approximately $1.6 \mathrm{~nm} / \mathrm{FWHM}$ and numerical aperture of approximately 0.16 . The sensor has no temperature stabilization or temperature measurement, thus the dark current is measured frequently during data acquisition and subtracted from the measurements. The

\footnotetext{
*riedel@geographie.uni-kiel.de; phone +49 (0) 8153281969
} 
spectrometer has been calibrated radiometrically at the RASTA radiance standard [8], which has an absolute uncertainty below $1 \%$ in the spectral range of $350-830 \mathrm{~nm}$. Additional to the radiometric correction a nonlinearity correction is performed.

The diffusor panel is a SRT-10-50 from the manufacturer Labsphere with a nominal reflectance of $10 \%$ and a surface area of $12.7 \times 12.7 \mathrm{~cm}^{2}$. The diffusor panel was calibrated in 2016 with a Perkin Elmer Lambda 1050 spectrophotometer for 2 different viewing directions. However a full BRDF measurement of the diffusor panel was not performed. Hence BRDF effects are not corrected for all measurements involving the diffusor panel. The measurement perpendicular to the diffusor panel, which is also the viewing angle of the spectrometer during measurement, was used to correct the measurements.

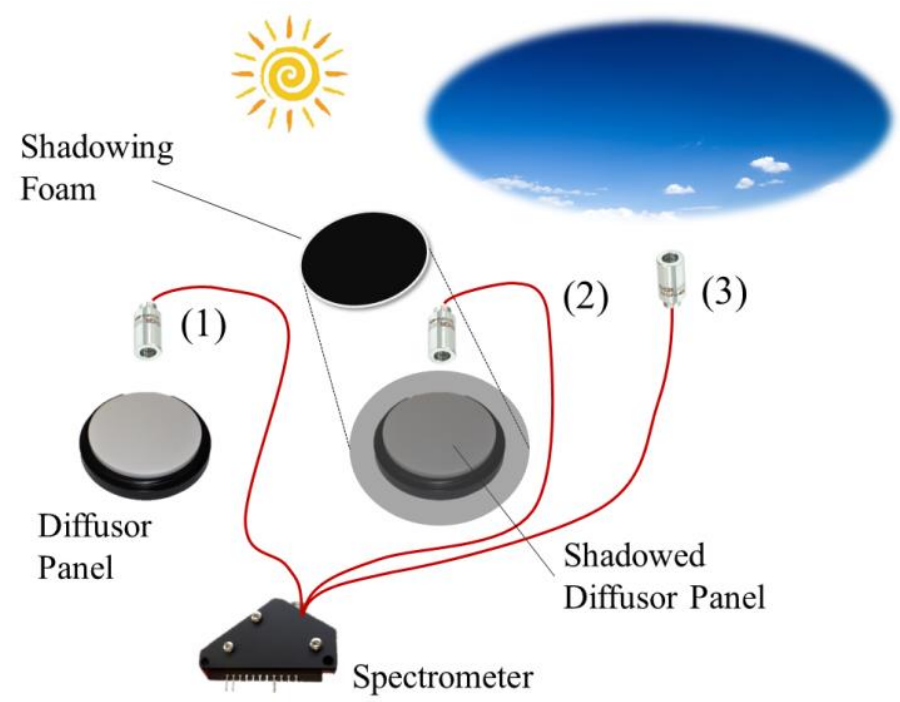

Figure 1: Schematic illustration of the used measurement concepts for measuring (1) total downwelling irradiance $E_{d}$, (2) diffuse downwelling irradiance $E_{d s}$ and (3) sky radiance $L_{s k y}$ in $0^{\circ}$ zenith angle direction.

The measurement principle for data collection is depicted in Figure 1. In total three measurements are performed in a rapid succession to avoid changes of the illumination conditions in between the measurements: (1) measuring the upwelling radiance reflected from the diffusor panel yields downwelling irradiance $E_{d}$. (2) Shadowing the diffusor plate from the direct sunlight and again measuring the radiance reflected from the diffusor panel gives the diffuse downwelling irradiance $E_{d s}$. (3) The sky radiance $L_{s k y}$ is measured in zenith direction.

To shadow the diffusor panel a circular piece of foam with a diameter of approximately $30 \mathrm{~cm}$ mounted on a $2 \mathrm{~m}$ long rod was used, which was held by hand and adjusted to minimize the area around the sun, which is covered additional to the solar disc. Therefore the covered area around the sun is unknown and variable during a measurement and in between different measurements.

\subsection{Theoretical Model and Data Fit}

A list of symbols and abbreviations can be found at the end of this paper (section 6).

The theoretical background for evaluation is provided by a model for irradiance on the earth surface, which has been developed by Bird and Riordan [9]. This model has been adapted to coastal and maritime applications by Gregg and Carder [10], and its spectral database has been refined to $1 \mathrm{~nm}$ resolution by Gege [11]. It is designed for clear sky conditions and in the current implementation adjacency effects are neglected [12].

Within this model the total downwelling irradiance $E_{d}$ is separated into contributions of the direct sunlight $E_{d d}$, diffuse part due to Rayleigh scattering $E_{d s r}$ at molecules, and diffuse part due to Mie scattering $E_{d s a}$ at aerosols

$$
E_{d}=g_{d d} \cdot E_{d d}+g_{d s r} \cdot E_{d s r}+g_{d s a} \cdot E_{d s a}
$$


The $g$ 's denote the intensities of the three light sources relative to unobscured sky and range from 0 to 1 . Blocking the direct sunlight is modeled by $g_{d d}=0$, and obstacles covering parts of the sky reduce $g_{d s r}$ and $g_{d s r}$ to values $<1$. The sky radiance is modelled similar to Equation (1) as a weighted sum of two spectrally different irradiance sources:

$$
L_{s k y}=l_{d s r} \cdot E_{d s r}+l_{d s a} \cdot E_{d s a}
$$

$l_{d s r}$ and $l_{d s a}$ denote the relative intensities of the radiances caused by Rayleigh and aerosol scattering, respectively. Since the angular distributions of Rayleigh and Mie scattering are different, it is $l_{d s r} \neq l_{d s a}$ and $g_{d s r} \neq g_{d s a}$ in general.

This model has been implemented into a Python program, which is used to extract atmospheric parameters from field measurements by fitting the model to measurements. Model inversion is performed by a nonlinear least-square minimization. Three different spectra are evaluated: the irradiance reflectance $R=E_{d s} / E_{d}$, the sky radiance reflectance $R_{s k y}=L_{s k y} / E_{d}$ and the sky radiance $L_{s k y}$. Reflectance measurements have the advantage that many instrument effects (except for signal nonlinearities) cancel out. Also, dividing two spectra with the same illumination conditions eliminates effects from absorbing atmosphere constituents. Adjacency effects cancel out completely if they are independent, and partially if they depend on the viewing direction. Using the parameterization of Bird and Riordan [9] for $E_{d d}, E_{d s r}$, and $E_{d s a}, R$ and $R_{s k y}$ can be expressed as

$$
\begin{gathered}
R=\frac{E_{d s}}{E_{d}}=\frac{\frac{g_{d s r}}{2}\left(1-T_{r}^{0.95}\right)+g_{d s a} T_{r}^{1.5}\left(1-T_{a s}\right) F_{a}}{T_{r} T_{a s}+\frac{1-T_{r}^{0.95}}{2}+T_{r}^{1.5}\left(1-T_{a s}\right) F_{a}} \\
R_{s k y}=\frac{L_{s k y}}{E_{d}}=\frac{\frac{l_{d s r}}{2}\left(1-T_{r}^{0.95}\right)+l_{d s a} T_{r}^{1.5}\left(1-T_{a s}\right) F_{a}}{T_{r} T_{a s}+\frac{g_{d s r}}{2}\left(1-T_{r}^{0.95}\right)+g_{d s a} T_{r}^{1.5}\left(1-T_{a s}\right) F_{a}}
\end{gathered}
$$

with

$$
\begin{gathered}
T_{a s}=\exp \left(-\omega_{a} \tau_{a} M(\theta)\right)=\exp \left(-\omega_{a} \beta \lambda^{-\alpha} M(\theta)\right) \\
T_{r}=\exp \left(\frac{M_{c}(\theta)}{115.6406 \lambda^{4}-1.335 \lambda^{2}}\right)
\end{gathered}
$$

where $M_{c}(\theta)$ is the atmospheric path length corrected for the actual pressure at the measurement site. Because $M_{c}(\theta)$ is only a function of the pressure and the sun zenith angle $\theta$ it can be calculated for a given location. Hence, $T_{r}$ can be calculated and requires no fit parameter in the retrieval. The aerosol scattering transmittance $T_{a s}$ contains the single scattering albedo $\omega_{a}$ and the atmospheric path length for standard pressure $M(\theta)$. $T_{a s}$ also contains the variables of interest, i.e., the turbidity coefficient $\beta$ and aerosol Ångström exponent $\alpha$ [13]. $\alpha$ and $\beta$ relate to the aerosol optical thickness $\tau_{a}$ as shown in Equation (7):

$$
\tau_{a}=\beta \lambda^{-\alpha} \text { with } \beta=\tau(550 \mathrm{~nm})
$$

The relative intensity factors $g_{d s r}$ and $g_{d s a}$ only contribute to $E_{d s}$ and not to $E_{d}$ due to the assumption that no part of the sky is covered during $E_{d}$ measurement. These parameters need to be determined during the retrieval, because the fractions of $E_{d s r}$ and $E_{d s a}$, which are cut away by shadowing the diffusor panel from the direct sunlight, are unknown. The sky radiance $L_{s k y}$, which is also measured, can be expressed within the model as

$$
L_{s k y}=\cos \theta_{s u n} F_{0} \cdot T_{a a} T_{o z} T_{o} T_{w v}\left(\frac{l_{d s r}}{2}\left(1-T_{r}^{0.95}\right)+l_{d s a} T_{r}^{1.5}\left(1-T_{a s}\right) F_{a}\right)
$$

The relative intensities $l_{d s r}$ and $l_{d s a}$, which depend on many parameters like sun zenith angle, measurement zenith and azimuth angle, aerosol parameters and air pressure, are fit parameters in the current implementation. Table 1 gives an overview on the fit parameters in the $R, R_{\text {sky }}$ and $L_{s k y}$ fits.

Time and location of the measurement are required as input to compute parameters, which are not fitted in the retrieval. These are used to calculate the sun zenith angle, which in turn is used to calculate the air mass [14] and forward scattering probability [9]. Also weather parameters such as relative humidity (which contributes to the single scattering albedo $\omega_{a}$ ) and pressure at the time of measurement (which contributes to the atmospheric path length $M_{c}(\theta)$ ) are required as input [9]. Relative humidity and pressure are automatically downloaded from a weather station server [15]. 
Table 1 shows an overview of fit parameters for all types of measurements.

\begin{tabular}{|c|c|c|c|c|}
\hline Symbol & Parameter & $\boldsymbol{L}_{\boldsymbol{s k y}}$ & $\boldsymbol{R}$ & $\boldsymbol{R}_{\boldsymbol{s k y}}$ \\
\hline$\alpha$ & Angström exponent & fit & fit & from $R$ fit \\
\hline$\beta$ & Turbidity coefficient & fit & fit & fit \\
\hline$H_{o z}$ & Scale height of ozone & fit & obsolete & obsolete \\
\hline$W V$ & Scale height of water vapor & fit & obsolete & obsolete \\
\hline$g_{d d}$ & Relative intensity of $E_{d d}$ for $E_{d}$ & 0 & 1 & 0 \\
\hline$g_{d s a}$ & Relative intensity of $E_{d s a}$ for $E_{d}$ & obsolete & fit & fit \\
\hline$g_{d s r}$ & Relative intensity of $E_{d s r}$ for $E_{d}$ & obsolete & fit & fit \\
\hline$l_{d s a}$ & Relative intensity of $E_{d s a}$ for $L_{s k y}$ & fit & obsolete & fit \\
\hline$l_{d s r}$ & Relative intensity of $E_{d s r}$ for $L_{s k y}$ & fit & obsolete & from $L_{s k y}$ fit \\
\hline
\end{tabular}

Fit results of the three types of measurements are combined to produce the best fit results. A schematic overview of the steps is shown in Figure 2. In the first step $L_{s k y}$ is fitted in the range of 350-500 nm, in the second step $R$ in fitted. The obtained values for $l_{d s r}$ and $\alpha$ serve as model constants for the $R_{s k y}$ fit to reduce the number of fit parameters. The fit range of $R$ and $R_{s k y}$ has been limited to $350-700 \mathrm{~nm}$, because the residual effects from $\mathrm{O}_{2}$ absorption and increased influence of instrument noise. The fit of $L_{s k y}$ is limited to $350-500 \mathrm{~nm}$, because this range is most sensitive to Rayleigh scattering.

In general it is possible to conduct measurements, which are suitable for aerosol retrieval with any spectrometer and diffusor panel. However, the spectral range of the spectrometer should be in the range where aerosol scattering has a significant impact on the downwelling irradiance.

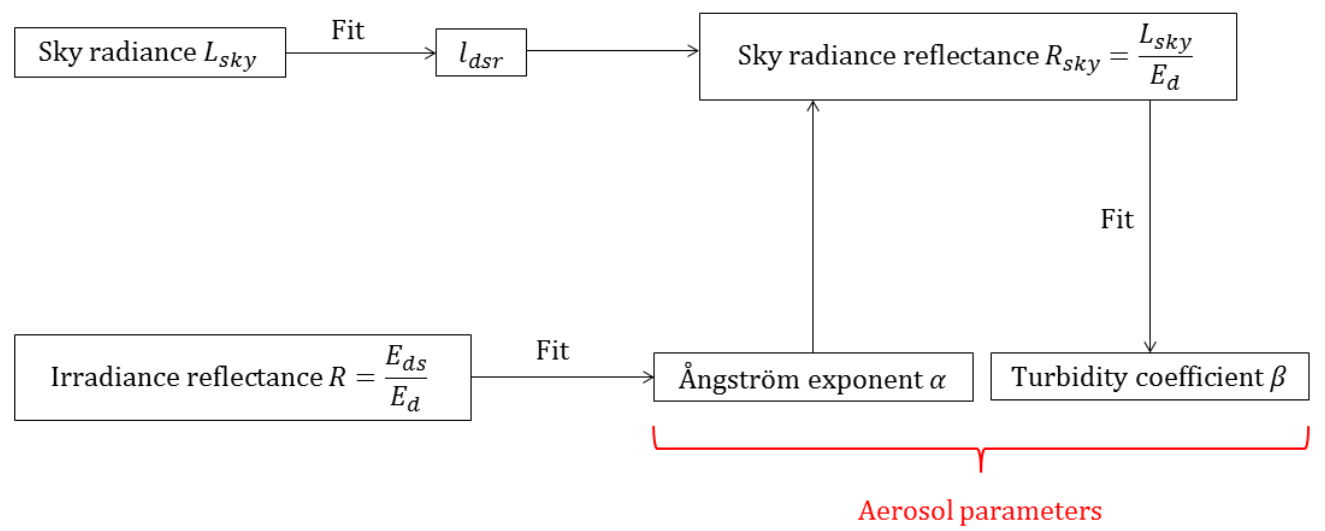

Figure 2: Schematic overview of the combination of all three measurements in the fit procedure The relevant fit parameters for the study goal are the aerosol parameters $\alpha$ and $\beta$.

\subsection{Data Collection and Validation Data}

Validation data for the fit results of $\alpha$ and $\beta$ were obtained from the AERONET station on the roof of the Meteorological Institute of the Ludwig-Maximilians-Universität (LMU) in Munich. AERONET provides values for the aerosol optical thickness (AOT) at multiple wavelengths and Ångström exponent values for multiple wavelength ranges. The turbidity coefficient is interpolated to $550 \mathrm{~nm}$ from AERONET AOT values for comparison with fit results by using Equation (7). 


\section{RESULTS}

Examples for measurement spectra from November $29^{\text {th }}, 2016$ (for brevity from now on called day 1) are shown in Figure 3. Measurements were performed on the roof of the Meteorological Institute of the LMU in Munich (geographical position: $48^{\circ} 08^{\prime} 52.0^{\prime \prime} \mathrm{N} 11^{\circ} 34^{\prime} 24.3^{\prime \prime} \mathrm{E}$ ) continuously in a time period of approximately 9:00-15:00 UTC in steps of 15 min. The distance to the AERONET station was approximately $20 \mathrm{~m}$, which is negligible. The three types of measurement were performed in rapid succession to minimize errors by changing illumination conditions. The weather conditions were cloudless during the whole day, with a very low aerosol load and a minimum zenith angle of $72^{\circ}$. Measurements were also performed on December $6^{\text {th }}, 2016$ (day 2) and February $16^{\text {th }}, 2017$ (day 3), also with cloudless conditions. The measurements show a significant influence of Fraunhofer lines (caused by absorbing constituents in the Solar and Earth atmosphere), which almost completely disappear after reflectance calculation.

The lower part of Figure 3 contains the irradiance reflectance and sky radiance reflectance calculated from the measurements above. The curves are very smooth except for a small residual effect from oxygen absorption at approximately $760 \mathrm{~nm}$ and some residual effects from instrument noise above $400 \mathrm{~nm}$. The curves have been scaled differently to better fit the plot boundaries.
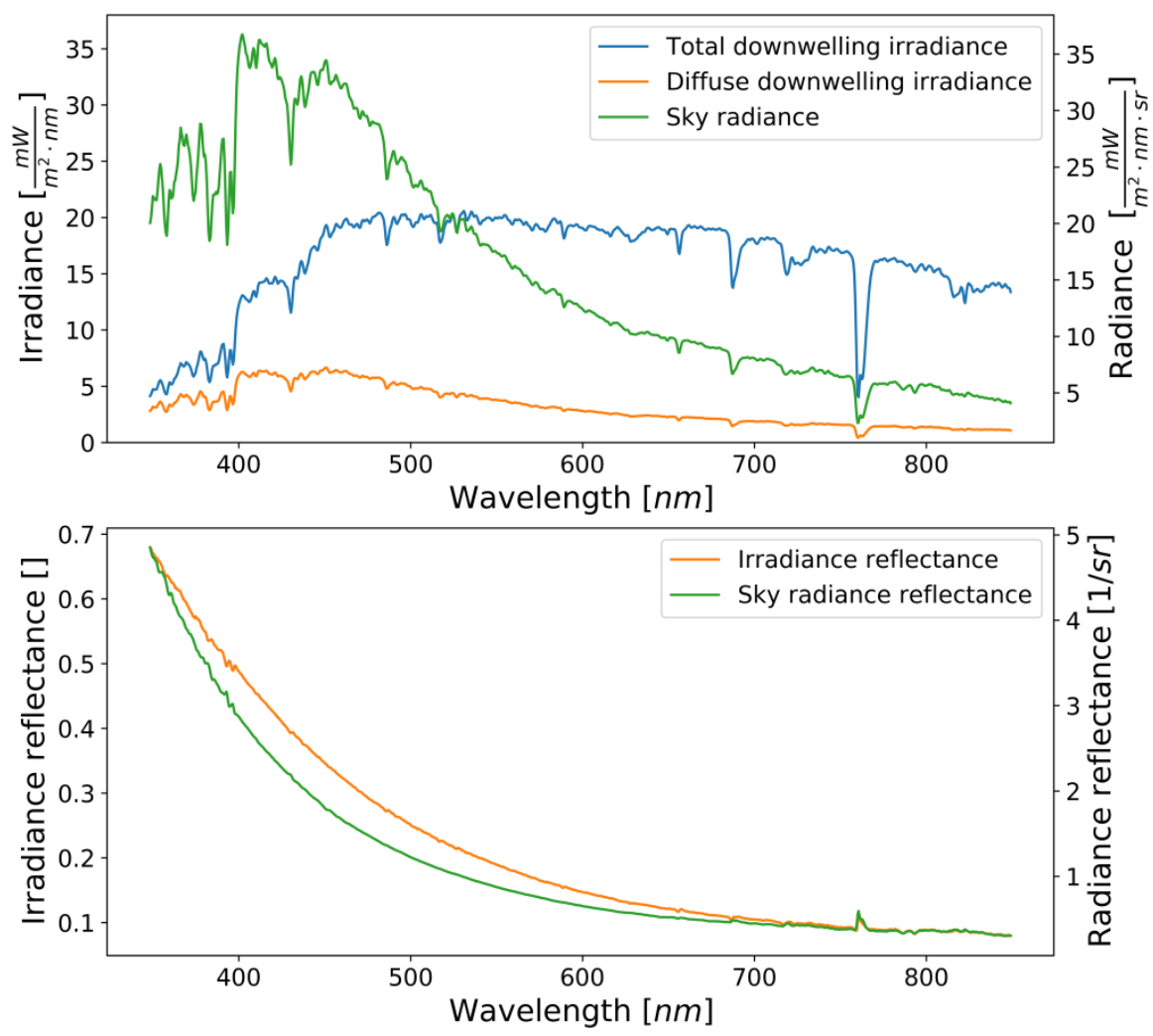

Figure 3: Top: example from November $29^{\text {th }}, 2016$ (day 1), for the three types of measurements used to calculate the spectra that serve as input for the fit model: total downwelling irradiance, diffuse downwelling irradiance and sky radiance. Bottom: reflectance spectra calculated from the three measurements above.

An extensive sensitivity study of the fit model revealed spectral ambiguities for different fit parameters, which introduce correlations between fit parameters [16]. The most problematic correlation was found between $\beta$ and the relative intensities $g_{d s a}$ and $l_{d s a}$. Due to these correlations not all spectrum fits yielded good results for all parameters. In the current implementation the model produces reasonable fit results for $l_{d s r}, \alpha$ and $\beta$, results for other parameters are often not reasonable. Results for $\alpha$ and $\beta$ will be further discussed in the results chapter. 
The redundancy of the three types of measurements is used to reduce the number of fit parameters for the $R_{s k y}$ and to improve the fit results. Figure 4 shows an example for fitting irradiance reflectance $R . \alpha$ is derived from this fit, and used as input parameter to the subsequent fit of sky radiance reflectance $R_{s k y}$ for the determination of $\beta$.

Figure 4 shows an example for a fit of the irradiance reflectance $R$ in the range of 350-700 nm. The fit reaches very good convergence and the fit residuals show little systematic error. The residual shows the largest deviation in the range of $350-400 \mathrm{~nm}$ due to the increase of detector noise in this range. However, the fit results show a dependency on the starting values, which need to be chosen in a realistic range for good results. The uncertainty of the retrieved parameters is derived from the fit covariance matrix and might not be comparable to the real uncertainty.

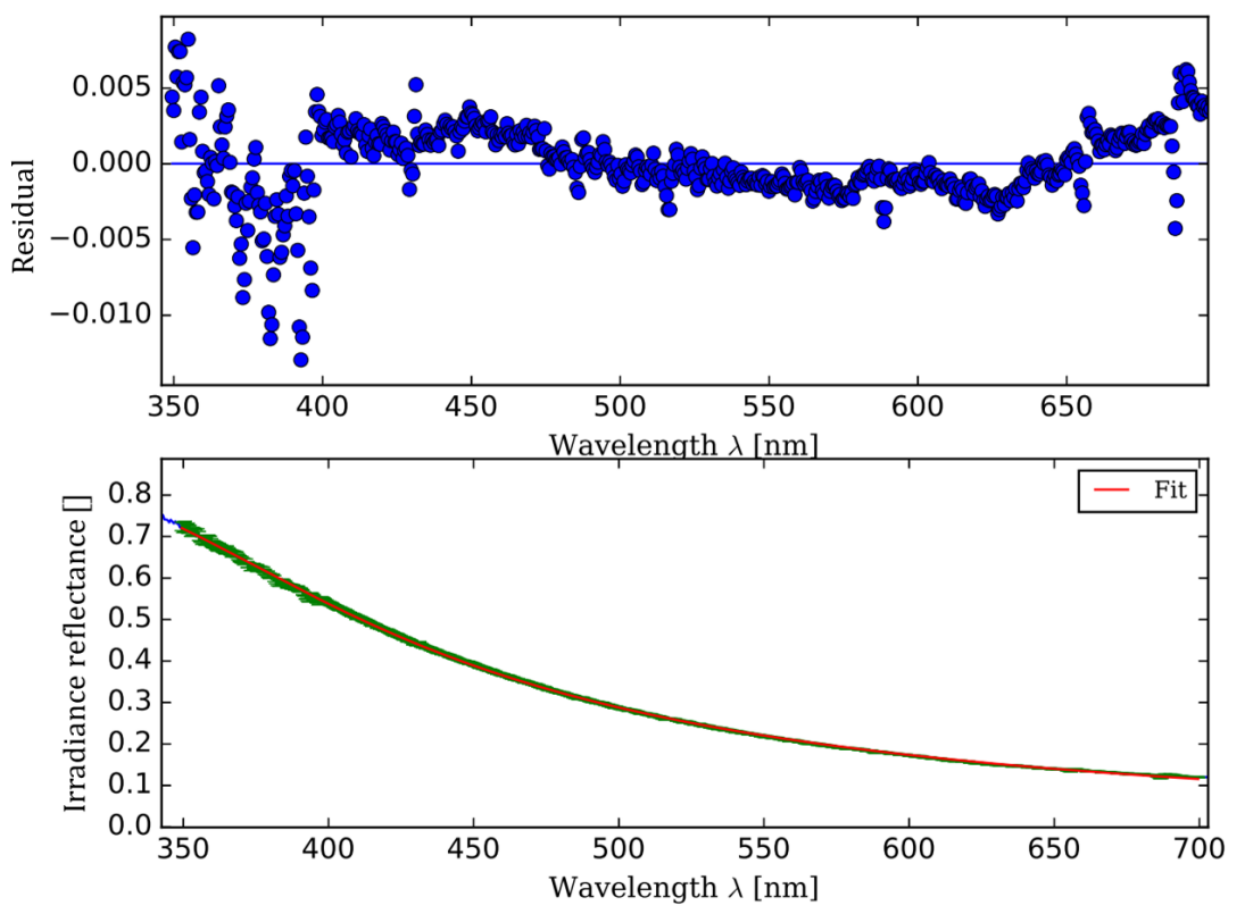

Figure 4: Example for the fit of irradiance reflectance $R(\lambda)$ (lower plot) and corresponding fit residuals in absolute numbers (upper plot). The fit does reach good convergence with little systematic error left in the residuals.

Figure 5 shows the fit results for $\alpha$ and $\beta$ in comparison to AERONET values for three measurement days. AERONET provides Ångström exponents for different wavelength regimes, which partially overlap with the fit range (350-700 nm). The three spectral ranges in AERONET, which overlap best with the fit range, have been chosen for comparison. The retrieved Ångström exponent is generally in the same range as the AERONET Ångström exponent but shows a different trend. The fit results from day 1 and day 2 look similar with best agreement with AERONET in the morning and a systematic decrease of the Angström exponent in the afternoon. This decrease is also present in the $\beta$ values.

The RMSE values calculated with the AERONET values as reference in absolute numbers for all days separately and in combination are given in Table 2. The RMSE for day 1 and 2 is in the range of $15-20 \%$ for $\alpha$ and 50-55\% for $\beta$. The fit results for $\beta$ exhibit a larger deviation from the AERONET values compared to the Ångström exponent deviation due to the correlation with $g_{d s a}$ and $l_{d s a}$. Also there is less systematic trend over time except for the systematic decrease for in the afternoon for day 1 and 2 . The results from day 3 exhibit a much larger deviation from the AERONET values compared to day 1 and 2 with a RMSE of approximately $40 \%$ for $\alpha$ and $900 \%$ for $\beta$. Also, the uncertainty from the fit covariance matrix is much higher for the $\alpha$ values, indicating that the fit was less stable. For this day the fit model was not able to produce useable results. 
29.11 .2016
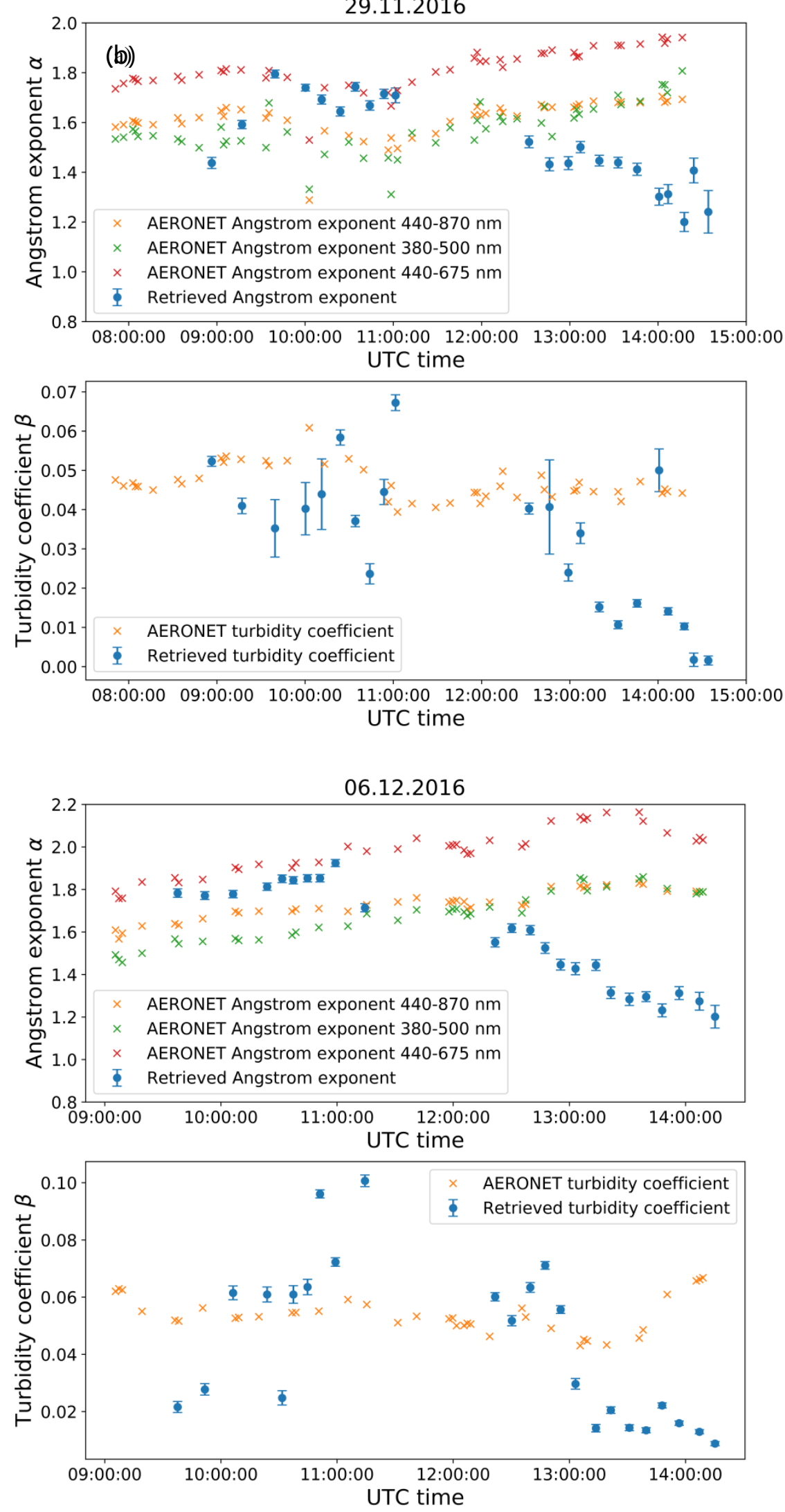

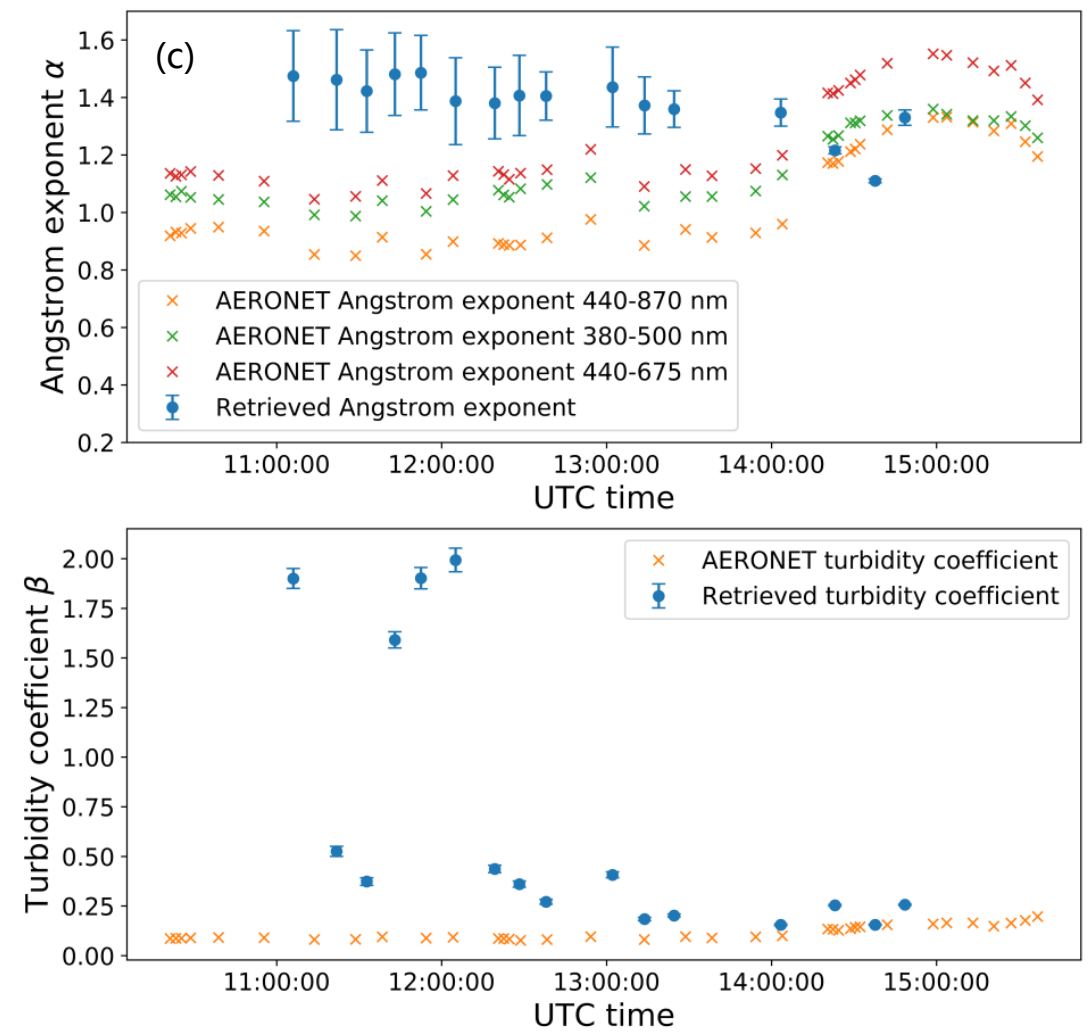

Figure 5: Comparison of the fit results for the Angström exponent $\alpha$ and turbidity coefficient $\beta$ with values from the AERONET station from (a) day1, (b) day 2 and (c) day 3. The $\beta$ values from day 3 are not realistic and have been excluded from Figure 6.

The correlation between the retrieved values and the AERONET values for $\alpha$ and $\beta$ for all days combined are shown in Figure 6. The $\beta$ values from day 3 have been excluded because of their large RMSE. The point clouds for day 1 and 2 show a trend close to an inverse correlation due to the fact, that the values decrease systematically in the afternoon, while the Ångström exponent measured by AERONET increases. Because of the large RMSE of the retrieved $\beta$ values, the distribution in the correlation plot is almost flat.

The linear regression for the Angström exponent shows a correlation of $R^{2}=0.035$ with $y=0.32 x+1.03$ and the regression for the turbidity coefficient shows a correlation of $R^{2}=0.036$ with $y=0.05 x+0.05$. This means that the fit results for $\alpha$ and $\beta$ are almost completely uncorrelated to the AERONET values. However, with only 3 measurement days there is not enough data to reliably find or exclude a correlation.

Table 2: RMSE values of the fit result with the AERONET values as reference for all measurement days. All RMSE values are given in absolute numbers. The $\beta$ values from day 3 have been excluded from Figure 6 and the combined value in this table (marked in red).

\begin{tabular}{|c|c|c|c|c|}
\hline Measurement & $\mathbf{2 9 . 1 1 . 2 0 1 6}$ & $\mathbf{0 6 . 1 2 . 2 0 1 6}$ & $\mathbf{1 6 . 0 2 . 2 0 1 7}$ & Combined \\
\hline $\operatorname{RMSE} \alpha$ & 0.27 & 0.34 & 0.47 & 0.36 \\
\hline $\operatorname{RMSE} \beta$ & 0.023 & 0.030 & 0.905 & 0.027 \\
\hline
\end{tabular}



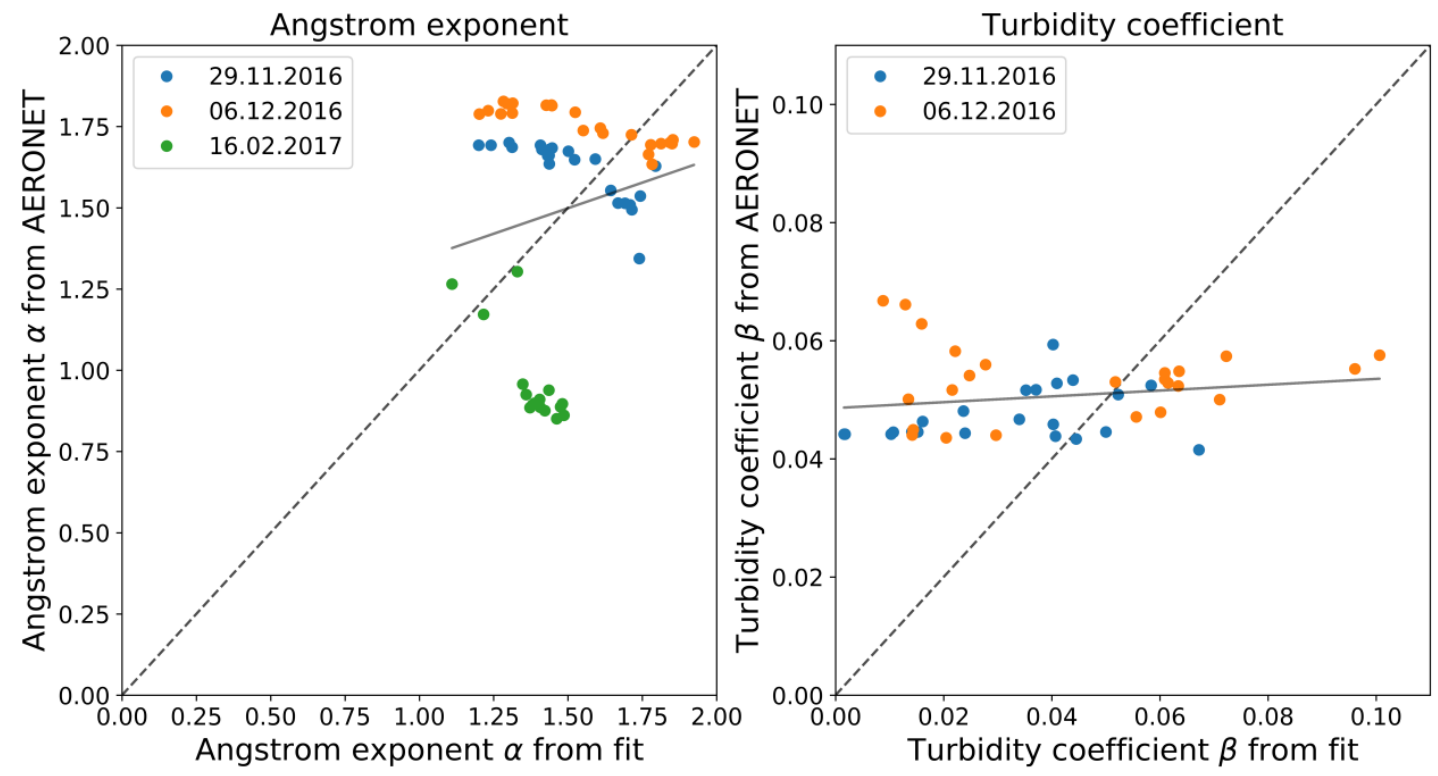

Figure 6: Correlation plots values for the retrieved Ångström exponent $\alpha$ and turbidity coefficient $\beta$ in respect to AERONET derived from the data shown in Figure 5. The fit result for $\beta$ on day 3 has been excluded from the plot.

\section{DISCUSSION}

The goal of this work is to develop a new method for extraction of aerosol parameters, which is applicable in conditions when it is impossible to measure with handheld sun photometers. The fit results for Ångström exponent $\alpha$ and turbidity coefficient $\beta$ are generally in the same range compared to AERONET values and even show good agreement in some cases, with exception of day 3 . After careful revision of the fit results and considering a minimum uncertainty of $15 \%$ (based on the current results), $\alpha$ values can be used for validation of atmospheric correction. However the fit results have a high dependency on the starting values for the fit and are limited in accuracy by the correlation between different fit parameters. Without more measurement data a correlation between the fit results and AERONET data can neither be proved nor excluded. Also, all measurements were performed in November, December and February, when zenith angles are high. It is currently unclear which effect causes the decrease of agreement between AERONET values and fit results towards the afternoon. The systematic decrease suggests that a parameter in the environment causes the problem in the fit. Further analyses would be required to investigate the influence of e.g. the sun zenith angle on the fit results.

One way to improve result accuracy is the implementation of a model for the relative intensities of sky radiance $l_{d s a}$ and $l_{d s r}$. This would reduce the number of fit parameters for $R_{s k y}$ and $L_{s k y}$ fits and should thus reduce the correlation between $l_{d s a}$ and $\beta$.

If there are multiple components in the aerosol size distribution, which usually is the case, these components have a varying influence on irradiance reflectance. When the diffusor panel is shielded from direct sunlight also a part of the sky radiance near the sun in cut away. Larger aerosol particles have a larger forward scattering component in the phase function than smaller particles, which introduces a bias into contribution of aerosol scattering to the diffuse downwelling irradiance $E_{d s}$. This results in an overestimation of $\alpha$. Therefore the goal is to use sky radiance reflectance $R_{s k y}$ for determination of aerosol parameters.

\section{CONCLUSIONS}

We developed a new method for extracting the turbidity coefficient and Ångström exponent of aerosols from groundbased spectrometer measurements. The method is functional in the current implementation, but has limitations, 
especially with the turbidity coefficient precision compared to AERONET data. With the current parameter quality the Ångström exponent values can be used for validation of atmospheric correction of satellite remote sensing data. Limitations arise from the correlation between the turbidity coefficient and other fit parameters. This correlation might be resolved by addition of a model, which describes the angle dependent contribution of aerosol and Rayleigh scattering. The uncertainty of the turbidity coefficient and the Angström exponent is expected to decrease significantly with this addition.

\section{LIST OF ABBREVIATIONS}

$E_{d}=$ Downwelling irradiance

$E_{d d}=$ Direct component of downwelling irradiance

$E_{d s r}=$ Diffuse component of downwelling irradiance due to Rayleigh scattering

$E_{d s a}=$ Diffuse component of downwelling irradiance due to aerosol scattering

$F_{0}=$ Extraterrestrial solar irradiance

$F_{a}=\quad$ Aerosol forward scattering probability

$g_{d s r}=$ Relative intensity of $E_{d s r}$ for $E_{d}$

$g_{d s a}=$ Relative intensity of $E_{d s a}$ for $E_{d}$

$\lambda=\quad$ Wavelength

$l_{d s r}=$ Relative intensity of $E_{d s r}$ for $L_{s k y}$

$l_{d s a}=$ Relative intensity of $E_{d s a}$ for $L_{s k y}$

$L_{s k y}=$ Sky radiance

$M(\theta)=$ Atmospheric path length for $1013.25 \mathrm{hPa}$

$M_{c}(\theta)=$ Atmospheric path length corrected for pressure at measurement location

$\theta_{\text {sun }}=$ Sun zenith angle

$\tau_{\mathrm{a}}=$ Aerosol optical thickness

$T_{i}=\quad$ atmospheric transmittance due to $r=$ Rayleigh scattering; $a s=$ aerosol scattering; $a a=$ aerosol absorption; $o z=$ ozone absorption; $o=$ oxygen absorption; $w v=$ water vapor absorption

$\omega_{a}=$ Single scattering albedo

\section{ACKNOWLEDGMENTS}

This work was conducted within the project KelpMap 2.0 (grant no: 50EE1351) funded by the Federal Ministry for Economic Affairs and Energy, Germany. 


\section{REFERENCES}

[1] S. Emberton, L. Chittka, A. Cavallaro, and M. Wang, "Sensor Capability and Atmospheric Correction in Ocean Colour Remote Sensing," Remote Sens., vol. 8, no. 1, p. 1, Dec. 2015.

[2] S. Sterckx, S. Knaeps, S. Kratzer, and K. Ruddick, "SIMilarity Environment Correction (SIMEC) applied to MERIS data over inland and coastal waters," Remote Sens. Environ., vol. 157, pp. 96-110, Feb. 2015.

[3] B. N. Holben et al., "AERONET -A federated instrument network and data archive for aerosol characterization," Remote Sens. Environ., vol. 66, no. 1, pp. 1-16, 1998.

[4] L. Harrison, J. Michalsky, and J. Berndt, "Automated multifilter rotating shadow-band radiometer: An instrument for optical depth and radiation measurements," Appl Opt, vol. 33, pp. 5118-5125, 1994.

[5] M. Morys, "Design, calibration, and performance of MICROTOPS II handheld ozone monitor and Sun photometer," J. Geophys. Res., vol. 106, no. D13, p. 14,573-14,582, Jul. 2001.

[6] Marian Morys, "Design, calibration, and performance of MICROTOPS II handheld ozone monitor and Sun photometer," J. Geophys. Res., vol. 106, p. 14,573-14,582, Jul. 2001.

[7] "Ibsen Photonics, https://ibsen.com/products/oem-spectrometers/freedom-spectrometers/freedom-vis/.".

[8] T. Schwarzmaier, A. Baumgartner, P. Gege, C. Köhler, and K. Lenhard, "The Radiance Standard RASTA of DLR's calibration facility for airborne imaging spectrometers," in SPIE Remote Sensing: Sensors, Systems, and NextGeneration Satellites XVI, Edinburgh, United Kingdom, 2012, vol. 85331U.

[9] C. Riordan and R. E. Bird, "Simple Solar Spectral Model for Direct and Diffuse Irradiance on Horizontal and Tilted Planes at the Earth's Surface for Cloudless Atmospheres," Sol. Energy Res. Inst., 1985.

[10] W. W. Gregg and K. L. Carder, "A simple spectral solar irradiance model for cloudless maritime atmospheres," Limnol. Oceanogr., vol. 35, no. 8, pp. 1657-1675, 1990.

[11] P. Gege, "Analytic model for the direct and diffuse components of downwelling spectral irradiance in water," Appl. Opt., vol. 51, no. 9, pp. 1407-1419, 2012.

[12] P. M. M. Groetsch, P. Gege, S. G. H. Simis, M. A. Eleveld, and S. W. M. Peters, "Variability of adjacency effects in sky reflectance measurements," Opt. Lett., vol. 42, no. 17, p. 3359, Sep. 2017.

[13] A. Angström, "Techniques of determinig the turbidity of the atmosphere 1," Tellus, vol. 13, no. 2, pp. 214-223, 1961.

[14] L. S. Murry, Physics of the Atmosphere and Climate. Cambridge University Press, 2012.

[15] "Weather station network, http://api.wunderground.com/.".

[16] J. Janas, "Extracting Atmospheric Parameters from spectrometer measurements and omparison with established methods," Master`s thesis, Friedrich-Alexander University Erlangen Nuremberg, 2017. 